\title{
Modeling of Multi-organization Performance for Emergency Response
}

\author{
Yufei Shu ${ }^{1}$ and Kazuo Furuta ${ }^{2}$ \\ ${ }^{1}$ RISTEX, Japan Science and Technology Agency, \\ ${ }^{2}$ Graduate School of Engineering, Tokyo University, \\ 100-0004 Tokyo, Japan \\ shuyufei@ristex.jst.go.jp
}

\begin{abstract}
To response severe disaster, such as hurricane, tsunami, or terrorists attack, multi-organization, such as government, companies, media, and public are involved. How to make these inter- or intra dependent organizations cooperate timely and accurately when they face uncertainty and stressful situation is the primary concerns. In this paper, we proposed multi-organization performance model, which introduced organizational factors, individual factors and focused on communication and collaboration among different organizations. Based on it we developed multi-agent emergency response system. This IT solution has capability to integrate drills into simulated response activities and reproduce the drills. To demonstrate how the system can be used as a powerful tool for supporting and evaluating the performance of multi-organization in disaster management and response, the test simulation is carried out using a scenario extracted from real-life emergency drills.
\end{abstract}

Keywords: Multi-organization performance, Multi-agent system, Emergency response, Disaster management.

\section{Introduction}

Statistics show globally, more than 2.5 billion people were affected by floods, earthquakes, hurricanes and other natural disasters between 1994 and 2003. A 60 percent increase over the previous two 10-year periods, U.N. officials reported. Not only protect from natural or man-made disasters, but also mitigate the effect when disaster occurs is recognized as most important way to save the society from losing life and property. Response to disasters in a timely and effective manner can reduce deaths and injuries, contain or prevent secondary disasters, and reduce the resulting economic losses and social disruption. Based on these considerations, emergency response systems are prepared before hand. Responding to large-scale disasters, such as sever hurricane, earth-quake, or terrorists attack it is a usual case that multiorganization involving many layers of government, commercial entities, media, and the public are involved. These entities work together to save lives, preserve infrastructure and community resource, and reestablish normality within the community. How to make these inter- or intra- dependent organizations cooperate 
timely and accurately in making critical decision when they confront uncertainties and stressful situations during crisis is the primary concerns.

As Klein (1997) claimed that human beings recognize the situation and make decision well under stressful situation if they have experienced the similar case. However, the experience of severe natural or man-made disasters is very less on hand. Because these kind accidents are rare happened and characteristics of risk are hardly to know. In addition, it is hardly to forecast how the multi-organization cooperatively response to severe disaster, because the multiformity of collaboration and diversity of communication among the organizations. The problem that emergency responders faced is that from those a few disaster experiences, it is hardly to develop proper emergency response system or assess the effectiveness of it that had already been developed. To enhance the capability of situation awareness, decision-making under crisis situation, emergency responders are trained to go through similar scene. One way is to conduct real-life drills. However, real-life drills are limited in many aspects. It is expensive in terms of time and resources. It is also hard to organize. Furthermore, detail planning is required to instrument drills. This makes it is almost impossible to test out response to the scenarios by "if-then" analysis. The other way is IT based solution, the computer simulation, which can help addressing many of the challenges brought forth by the need for emergency response preparedness. It is much more flexible and can test various responses by setting different simulation scenarios. As US Department of Defense (DoD) found that use of simulations instead of live exercise for training can reduce the training cost to one-tenth (Jain and McLean, 2003). It is recognized as a good alternative and complementary method to training responders and assessing performance of emergency response system.

A numbers of emergency response simulation system have been developed (Cohen, etc., 1989; Avesani, etc., 2000; NSSL, 2003; LANL, 2003; A-TEAM, 2005). By reviewing the state of the art, we noticed that major parts of the emergency response studies focus on the model disaster itself, for instance, the fire dynamics inside buildings. What is not studied in as much detail is modeling response activity, especially modeling response activity of inter, intra-dependent organizations. For the emergency response, not only we need to know how the disaster propagate, how to evacuate, but also we should find out how can effectively cooperate between different organizations because it plays heavy role to mitigate the effect of disaster. When the severe disaster occurs, multi-organization cooperate and coordinate to deal with emergency situations. It becomes very necessary to model and assess the performance of cooperated multi-organization. Although it is important, how to model multiorganization performance, how to assess that if the emergency response system is well designed is still not established well.

Our study will explore the design of organizational models for emergency response to increase the efficiency of entities organizations under the conditions of uncertainty and rapid change. Therefore, the aim of this study is firstly to understanding the underlying mechanism of multi-organization cooperation and to propose a multiorganization performance model, which will focus on communication and collaboration between different organizations. Since the real socio-technological system is so complex, the models need to be designed to abstract the critical points of interaction within each organization, as well as the critical points of interaction among the organizations. Secondly, based on proposed team model, we build a parallel and 
distributed multi-agent system to simulate emergency response activity of multiorganization, that agents play different roles to drive simulation. In addition, various modules are embedded in the system to capture information, make decisions, and execute action.

\section{Multi-organization Performance Model}

\subsection{Multi-organization Cognitive Process}

There are numbers of research efforts have been targeted at modeling small team behavior in the context of specific application domain, such as industrial process control, aviation, or ship navigation, etc. For example, Furuta and Shimata (1999) analyzed team cognitive process in open and interactive style. Milici (1996) used fixed coordination pattern to study team performance. Shu etc. (2002) modeled team behavior in a dynamic scenario to simulate operator team response to accident in nuclear power plant. Shu etc. (2005) studied team situation awareness and developed criteria to assess it. These works have been proved successfully reflect the essential characteristics of team cognitive process in engineering application domain. However, since man-made disaster risk has increased due to a possibility of terrorist attacks in the world wide. Also, statistics shows that natural disasters also have increasing intention. The analyzing of team performance has been shifted to the context of social-technology system, especially, multi-organization participating system for emergency response. Analysis sophisticated multi-organization performance, the multiformity of collaboration and diversity of communication within organizations should be toke into account.

Considering the fact, that members of large team can be divided into smaller groups or arranged in a hierarchical structure, we will analysis multi-organization performance based on our previous research on small team behavior. We have proposed TBNM model using a serial sub-model to describe context of team performance (Shu, etc. 2002). The model defined task structure, event propagation, team factor, and individual factor, which determine the basis of communication. In this study, we adopt this TBNM model to describe multi-organization performance. We analyze multi-organization cognitive process within a hierarchical organizational structure, because it is a usual case within sophisticated social-technical system. In the multi-organization, since assigned tasks are different among the organizations, emergency response task is carried out via communication and collaboration. Multiorganization's emergency response process consists of recognition of information, decision-making, planning and execution as shown in Fig.1. The collaboration or communication scheme is not static due to changing of environmental situations. It is vitally affected by social interaction. Organization or member judge, decide, plan, and act based on the knowledge they have and the information they obtain from the environment and other members. Collaboration and information exchange among organizations or members are accomplished through communication and embedded in the cognitive process. It is triggered by task structure, organization structure, individual factor (e.g. distribution of knowledge, authority among members, etc.). This team-oriented cognitive process emphasized that problem solving is 


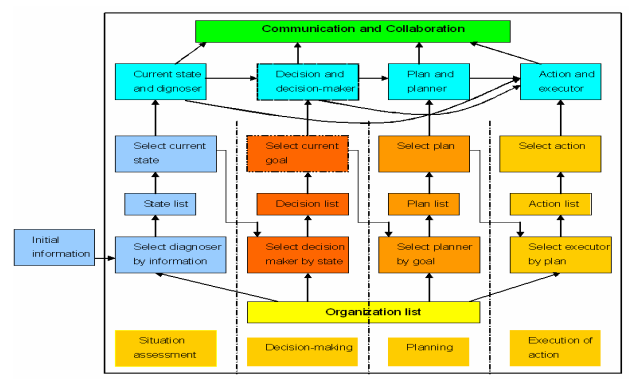

Fig. 1. Multi-organization cognitive process

accomplished through communication and coordination between members. It is domain independent, which can describe the multi-organization behavior from small operator teams to local government offices.

\subsection{Role-Based Agent Model}

In the hierarchical multi-organization, each organization or member play different role to carry out the emergency response task. The organization or member is modeled as a rule-based human agent embedded in the team cognitive process with input-recognition-decision-plan-action cycle. Depending on the concrete simulation requirement, agent can be designed according to the scale of participants and granularity of organizations by configuring knowledge base for each organization.

An agent obtains information or resources from the environment and others agents, recognizes situation, decides, plans referring to the knowledge base, and then acts, as shown in Fig.2. We predefine the profile of each agent, including name of agent, position in the organization, resource they equipped, contact point with other agent, individual factors such as knowledge, duty, and authority distribution among members over the organizations, and emergency responding procedures. Individual factors contribute to determining path of communication. The distribution of knowledge defines knowledge about emergency response task that each agent has. The distribution of authority defines authority to make a decision that each agent has. Although some organizations know how to execute the action well, they are not allowed to make decision of execution of certain action, if they do not have authority to do so. Distribution of authority is used to find an appropriate decision-maker among the cooperative organizations. Distribution of duty means that each agent takes the different charge of execution. It used to find an executor of action through the entity of organizations.

Disaster information and resource as initial information are input to the agent model. Resources that each agent equipped are defined initially. According to the different disaster drill setting, we can design different contents and allocation of resources that each agent holds. These resources, such as workforces, telephones, fax, are required for executing certain actions. When agent uses resource to execute actions, the number of resources decreases. When agent obtains a new resource message, the number of resource increases. 


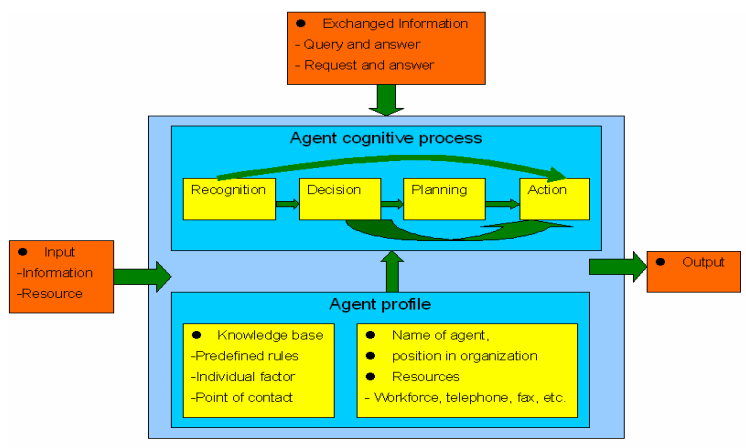

Fig. 2. Agent model

When the new message is received, based on the contents of message, agent recognizes the current situation and appropriate decision are determined in this step. Since emergency response is shaped in light of individual factors, such as distribution of authority, duty and knowledge, primitive response can be determined by referring knowledge base. However, some responses are depending on the situation. These responses are determined by experience. Rules and protocols used in this study were extracted from the emergency drills and result of interviews emergency responders.

After decision made, a typical and important response plans or actions should be decided, which were constructed by goals-means task analysis (GMTA). They are prescribed in knowledge base extracted from emergency response procedures or manuals, such as resident evacuation, dispatch fire-fighter forces or star emergency transportation. Procedure is series of actions, it consists preconditions and detailed actions. Each action requires certain preconditions, resources, time period, and effects. When preconditions satisfied, the action will be initially automatically.

Usually, cooperation and communication among organizations are required to accomplish emergency response task. It is achieved by exchanging message among agents. A message contains various type of basic data used for representing information and resource exchanges in the real situation, such as information sender, receiver, time and contents of communication. We classified the verbal protocol (utterances) using the message type extracted from drills and used them in the simulation system. The utterance includes inform, query, and request. ACL-based (Agent Communication Language) messages transfers are used in this study for exchanging information. The disaster-related information including contents of communication between agents is storied in the messages storage.

\section{Multi-agent Emergency Response System}

Emergency response system for dealing with nuclear disaster had been developed in our previous work (Kanno and Furuta, 2004). To enhance the ability of handling large spectrum of disaster, such as natural or man-made disaster, the system is implemented under various situations, especially, applied to various social-technological systems. The domain independent proposal is developed to handle different types of crisis and 
complete different emergency response tasks. Since multi-organization usually use synthetic environments for mission rehearsal exercises, training, or testing the response plans. We are developing multi-agent simulation system based on the above model to reproduce the synthetic environments and represent multi-organization emergency response in it. The simulator mimics the emergency response activities of multi-organization and how each organization interacts with other organizations. Each organization is modeled as an agent that can receive and recognize information obtained from the environment and other agents as well as make decision based on its own knowledge. Each agent is assigned a role for accomplishment of entity emergency response task. Communication and coordination between agents are modeled as information exchange. In order to achieve full potential as a supporter and assessor of emergency response system, the simulator is constructed as a knowledge base system with different functional capabilities. The architecture of it consists of several different technical modules: team cognitive process module, agent cognition module, disaster scenario module, interface module, communication channel, and knowledge base, as shown in Fig.3. The system is developed in program language $\mathrm{C}++$ and Java.

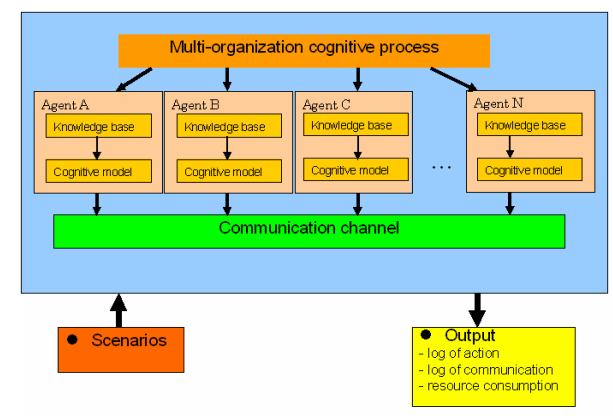

Fig. 3. Architecture of emergency response system

Multi-organization cognitive process module: describes the process of how multiorganization accomplish emergency response task via coordination and communication. It provides a platform to describe disaster propagation, emergency response task, behavior of each agent, and communication paths.

Agent cognition module: this module simulates behavior of organization or member embedded in the disaster response process, such as police, fire fighter, medical staff, or state government agencies. Depends on the scale of simulated disaster, the scale of agents and granularity of organization can be a small group or city or state government offices. The profile of each agent is prescribed, such as name of the agent, predefined responding procedures (e.g. precondition, action), individual factors (e.g. authority, duty), contact point to the other agent (e.g. where or whom the information should sent to). Resources (e.g. phone, fax, and car) of each agent are described in the resource storage. The contact point and responding procedures are extracted from real-life drill and interview with disaster responder. At any given time, agents are associated with a given task in the disaster response synthetic environment. 
Communication channel: this module provides platform for information exchange among the agents. In our study, we use CORBA (Common Object Request Broker Architecture) to transfer information. An agent was implemented as a distributed object constructed on the CORBA platform. Message exchange among the agents is carried out via CORBA APIs.

Scenario module: This module describes the disaster-related information, such as event propagates, information exchange among the agents. Scenario stores sets of messages corresponding to the real-life drill scripts that are used by the drill participants. We represent scenario as a snapshot taken at every time unit. This snapshot is reproduced as a set of information which includes information sender, receiver, time, contents of the information, etc. It is used as inputs of the simulation system and then delivered to the proper agent according to the contents of message. Interface module: The interface allows that users interact with the simulation environment and set input to the simulator. The user can start the simulation, select the response members, chose the granularity of organizations through the interface. The outputs of simulation system are logs of communications, actions, and resource consumptions for each agent. By analysis these simulation logs, the process of multiorganization collaboratively response to the emergency and the performance of the multi-organization can be evaluated and visualized via GUI. The GUI is developed in a programming language Java.

\section{Emergency Response Simulation}

The most important aim of this IT solution is to support and evaluate emergency response system. To demonstrate how this IT solution can be used as a powerful tool for supporting and evaluating the performance of multi-organization in disaster management and response context, the test simulation is carried out. Firstly, we represent the real-life drill through the computer-based simulation. Secondly, we test the response procedure under different drill settings and assess the emergency response system by changing design of agent profiles, or structure of organizations.

\subsection{Simulation Scenario}

Since there is high probability of sever earth quake in Kanto area of Japan in the future 50 years, the major Japanese estate company conducts real-life drill every year to train staffs responding to sever earthquake. The drill role assignment and drill scripts that they delivered to the real-drills participants are used in our test simulation.

According to the drill settings and scripts, the simulation scenarios are extracted and profiles of agents are predefined. The emergency response system is divided in to five hierarchical agent groups: headquarter, information section, administration and advertising section, restoration section, and owners. Each group consists of number of agents in a hierarchical structure, equipped with fixed phone, satellite phone, fax, and PC, etc. Agent profiles, such are individual factors (e.g. role, authority, duty, etc.) and resources (e.g. equipment, workforce, etc.) were designed in light on the drills scripts. The knowledge base of each agent is extracted from the earthquake response procedures and manuals. When the disaster message is received, all agents have to 
work cooperatively to judge the situation, and make corresponding decision. Communication and cooperation paths among the agents are determined according to drills. Real-life drill was conducted in 2 days, totally costing 2 hours and 20 minutes. We will reproduce it on the computer based simulation system within 2 hours and 20 minutes. When we change the contents of agent profile, or organizational structure, we can analyze emergency response system under different settings. In this test application, two groups of simulations setting are prepared by changing agent profiles. One situation is that authority is centralized at headquarter group, and the other is de-centralized among different agents.

\subsection{Testing Results and Discussions}

Outputs of simulation are $\log$ of communication and action of each agent, and resource that each agent used. The response activity of entity organization is shown in Fig.4. This figure is a combined form of the communication flow diagram and the action flow diagram of multi-organization. In the figure, $\mathrm{x}$ axis represents time flows from left to right. Communication between agents includes information and resource exchange, e.g. call, fax, etc. is represented as an arrow, while the no-communication actions, such as meeting, setting, etc. corresponds to the colored lines labeled with the action name. Each horizontal line donated to the agent or agent group. When organization model described in agent profile has been changed, as expected, the performance of multi-organization is changed accordingly.

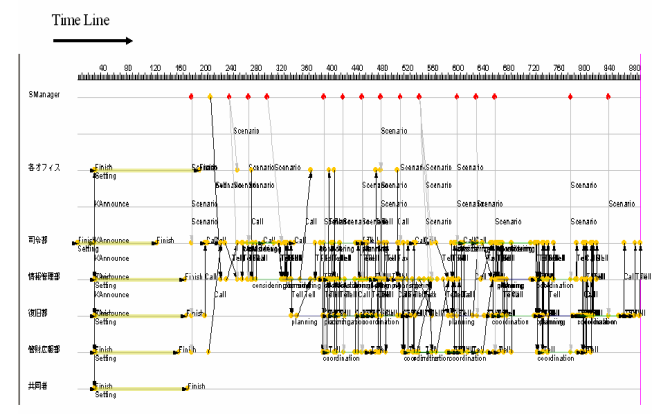

Fig. 4. Task and cognitive action flow

We analyze the outputs of simulation under different settings. Fig.5 and 6 shows the workload at headquarter, administration section, and information section under different simulation settings respectively. Here, workload refers to the number of active and queue actions per head in the group. We can notice the workload is changed accordingly as expected. By distributing the authority of decision-making, the workload on headquarter agent is decreased specifically. We also can see that workload on the advertising group is higher, due to it is required to deal with gather information, report to headquarter, and executing certain tasks. 


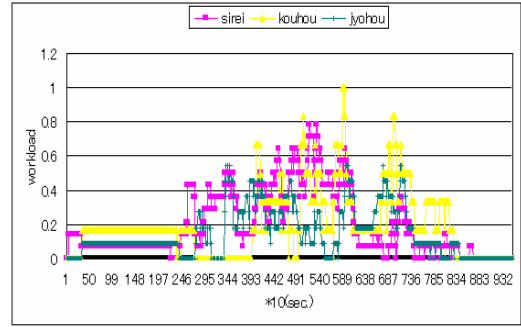

Fig. 5. Authority is centralized at headquarter section

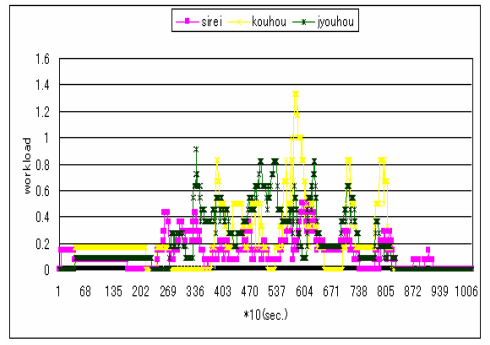

Fig. 6. Authority is decentralized in each section

By represent the real-life drill, we acquire insights for effective emergency response. For example, we can find the bottleneck of coordination in the drill scenario or identify the vulnerability of emergency response system. We also can see the workload at the high level of hierarchical organizations tended to be accumulated because of limitation of information process capacity. It indicates that emergency response system should be designed according to the capacity of processing information.

Through the simulation, we investigated how cognitive process and workload of each agent changes corresponding to the change of emergency response system. Through analyzing the simulation results, we can evaluate the efficiency of the emergency response system and optimize the structure of emergency response system, resource distribution and response procedures, manual.

\section{Conclusions}

In order to support and assess various emergency response systems, we have proposed rule-based multi-organization performance model, which focused on the cooperation and communication among organizations. Based on the proposed model, we have developed organizational activity simulator by taking earthquake disaster example. The test simulation was carried out and results were compared with real-life drills. Through the test, we confirmed that model reflects the multi-organization behavior very well and the computer based simulator has capability to represent the real-life emergency response drills and to evaluate emergency response system.

Nevertheless, since our model simplified the complex real environment, the model has limitation. The integrated simulation model requires that data transfer from one sub-model to another in the right format. Building the library of objects requires a large effect for simulator implement in the disaster context. This type or simulator, however, in the context of emergency management, is suitable for training emergency responder involved in the disaster by supplying "if-then" scenarios. Also, it can provide various criteria for evaluating performance of existent emergency response system and optimizing organization structure, response procedures or manuals.

Acknowledgments. Work described in this paper was sponsored by Japan Science and Technology Agency. 


\section{References}

1. Avesani, P., Perini, A., Ricci, F.: Interactive case-based planning for forest fire management. Applied Intelligence 13, 41-57 (2000)

2. A-TEAM (2005) http://www.ess.co.at/A-TEAM/

3. Cohen, P.R., Greenberg, M.L., Hart, M., Howe, A.E.: Trial by fire: Understanding the design requirements for agent in complex environments. AI Magazine 10, 32-48 (1989)

4. Furuta, K., Shimada, T., Kondo, S.: Behavioral simulation of a nuclear power plant operator crew for human-machine system design. Nuc. Engne and Desg. 188, 97-109 (1999)

5. Jain, S., Mclean, C.: A framework for modeling and simulation for emergency response. In: Proc. Winter simulation conference 2003, pp.1068-1076 (2003)

6. Kanno, T., Furuta, K.: Multi-agent simulation of emergency response in nuclear disaster. In: Proc. Int'l Conf Probabilistic safety assessment and management (PSAM7), pp. 389-394 (2004)

7. Klein, G.: The Recognition-primed decision (RPD) model: looking back, looking forward, Naturalistic decision making, pp. 285-292. Erlbaum, Nahwah (1997)

8. LANL, 2003. Interdependent infrastructure modeling, simulation, and analysis project (SOFIA) http://www.lanl.gov/orgs/d/d4/infra/sofia.html

9. Milici, A., Wu, S.J., Apostolakis, G.: The use of the dynamic flowgraph methodology in modeling human performance and team effects. In: Proc. Int'l Conf Probabilistic safety assessment and management (PSAM3) pp. 1-6 (1996)

10. NSSL. National severe storms laboratory's warning decision support system (2003) http://www.nssl.noaa.gov/wrd/msd/wdss.html

11. Shu, Y., Furuta, K., Kondo, S.: Team performance modeling for HRA in dynamic situations. Reliab Engne Syst. Safety 2002 78, 111-121 (2002)

12. Shu, Y., Furuta, K.: An inference method of team situation awareness based on mutual awareness. Cogn, Tech. and Work 7, 272-287 (2005) 